\title{
Government could help prison TB victims - activists
}

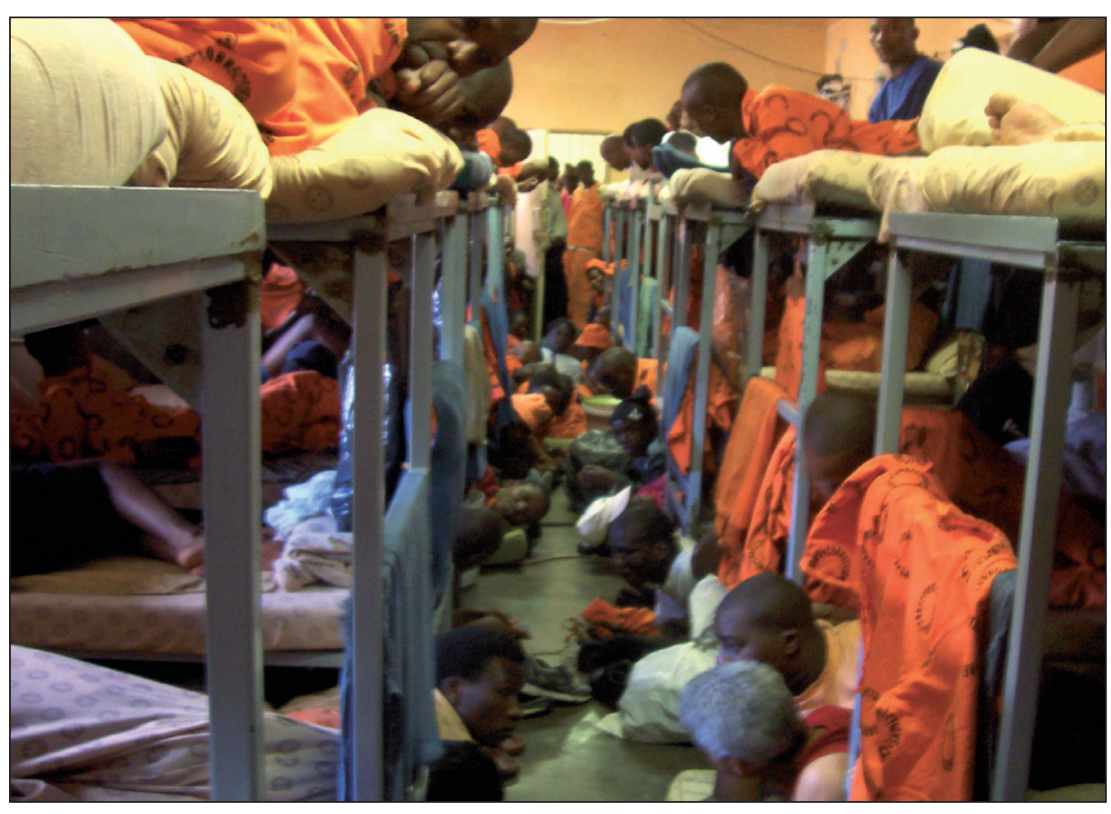

South Africa's overcrowded prisons are massive TB breeding grounds but there is 'little political will' to prioritise interventions which a joint university study in the Western Cape shows could reduce transmission by up to $94 \%$.
Simply implementing existing maximum cell occupancy regulations would reduce TB transmission by $30 \%$, while active case finding, ventilation and reduced time in cells would do the rest, researchers at the universities of Cape Town and
Stellenbosch claim. ${ }^{1}$ The study was cited by four internationally respected healthlinked activist organisations in a joint declaration on the eve of the third national bi-annual TB conference in Durban (12 15 June).

South Africa has the fourth highest global incarceration rate, with more than 165000 prisoners in 237 operational prisons. There is a rapid turnover of awaitingtrial prisoners with $79 \%$ being incarcerated for periods of less than 12 months. The number of individuals passing through the prison system annually therefore exceeds 368000.

The Treatment Action Campaign, Section 27 (incorporating the AIDS Law Project), Médecins sans Frontières (MSF) and Oxfam (the international poverty relief confederation) said the university study 
exposed a $90 \%$ probability of TB transmission per patient per year in Pollsmoor, a large prison in Tokai in the Cape Peninsula. Overcrowding was a major infection driver, with prisoners commonly held in mass cells in extremely close proximity for up to 23 hours a day. Besides the obvious measures, the departments of correctional services and health should ensure that prisoners who were TB-positive were diagnosed earlier and received proper treatment to reduce mortality. Pollsmoor mirrors disease conditions in scores of other South African prisons.

South Africa has the fourth highest global incarceration rate, with more than 165 000 prisoners in 237 operational prisons. There is a rapid turnover of awaiting-trial prisoners with $79 \%$ being incarcerated for periods of less than 12 months. The number of individuals passing through the prison system annually therefore exceeds 368000 . A 2006 Judicial Inspectorate of Prisons report showed prisoner mortality in the country rose from 1.65 deaths/ 1000 prisoners in 1995 to 9.2 deaths/ 1000 prisoners in 2005, a 5 -fold increase. ${ }^{2}$

\section{Decentralise drug- resistant $\mathrm{TB}$ care - and fast}

If the country's leading cause of death was to be reduced, it was 'crucial' that there be a general expansion of TB diagnosis and access to existing and improved treatment for TB and multi-drug-resistant TB (MDRTB). Services could no longer be limited to certain areas and facilities while better diagnostic tools and medicines and access to them were 'vital', the organisations said. Five crucial interventions were needed: diagnosing all people living with $\mathrm{TB}$ and drug-resistant (DR) TB; ensuring access to the best available medicines and regimens; improving the affordability of medicines; decentralising DR TB care; and reducing prison crowding and implementing active case finding and infection control. Calling for decentralised care to be rolled out nationally via the training of nurses to manage treatment and community health workers to support ongoing care, the group acknowledged the government's policy shift to decentralisation but said the handful of current sites was simply not enough.

'Nearly half of patients diagnosed with DR TB in South Africa are not initiated onto treatment and with only 2500 beds for DR TB patients, centralised care is no longer possible or cost-effective,' they said. One (unnamed) decentralised pilot site in KwaZulu-Natal had improved cure rates by $66.7 \%$ (versus the last recorded national DR TB treatment success rate of $48 \%$ in 2008). At Tugela Ferry District Hospital (where extensive MDRTB was first uncovered by its Principal Medical Officer, Dr Tony Moll 6 years ago), active case finding, decentralised care and infection control had decreased the rate of new MDR infections by half. (Ironically, the international publicity generated by Moll's initial pragmatic sleuthing has generated funding and equipment for his hospital that is the envy of its peers.)

At Tugela Ferry District Hospital (where extensive MDRTB was first uncovered by its Principal Medical Officer, Dr

Tony Moll 6 years ago), active case finding, decentralised care and infection control had decreased the rate of new MDR infections by half. (Ironically, the international publicity generated by Moll's initial pragmatic sleuthing has generated funding and equipment for his hospital that is the envy of its peers).

\section{Hard bargaining/ creativity needed on medicine prices}

The activists said that while the Department of Health had reduced the prices of several TB medicines during its last tender, the country continued to pay higher prices for many medicines than elsewhere internationally. Linezolid, for example, was unaffordable and should be available at lower costs. Pfizer charged R8 460 per patient per month (for linezolid) in the public sector and more than double this for NGOs such as MSF. Suggested solutions included importing lower-cost medicines from overseas or pooling procurement with other high TB burden countries. Funders and developers of new medicines in the pipeline should ensure that, once registered, they were made 'widely available at low costs'.

The group lauded the introduction of the GeneXpert diagnostic tool by Cepheid (able to detect TB in many patients who currently test false-negative using smear microscopy plus detect rifampicin resistance), but criticised the cost of the machines and cartridges, saying a cost reduction would allow critically needed expanded use in all developing countries. More research was needed to discover cheap, laboratory-free, point-of-care diagnostics. Linezolid offered hope for patients failing on DR TB but was not yet widely available. The compassionate use of bedaquiline (promising results in Phase II trials) for patients who had no other treatment options provided a potential chance of cure and was already conditionally available in several countries. The group called for the Department of Health and the Medicines Control Council (MCC) to facilitate access to important new medicines in the pipeline for patients in dire need. Once these medicines received pre-approval from the Food and Drug Administration (FDA), they should be fast-tracked by the MCC. The group said funding for research into new medicines to shorten and simplify regimens and reduce side-effects (hearing loss, paranoia, depression and kidney failure) was critically needed. (TB regimens usually take 6 months to complete and DR TB regimens up to 2 years).

\section{Chris Bateman}

chrisb@hmpg.co.za

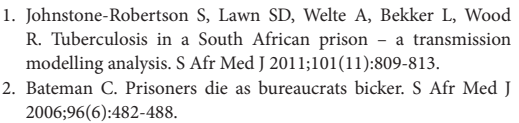

1. Johnstone-Robertson S, Lawn SD, Welte A, Bekker L, Wood R. Tuberculosis in a South African prison - a transmission modelling analysis. S Afr Med J 2011;101(11):809-813.

Bateman C. Prisoners die as bureaucrats bicker. S Afr Med J 2006;96(6):482-488.

S Afr Med J 2012;102(8):645-646.

DOI:10.7196/SAMJ.6082 